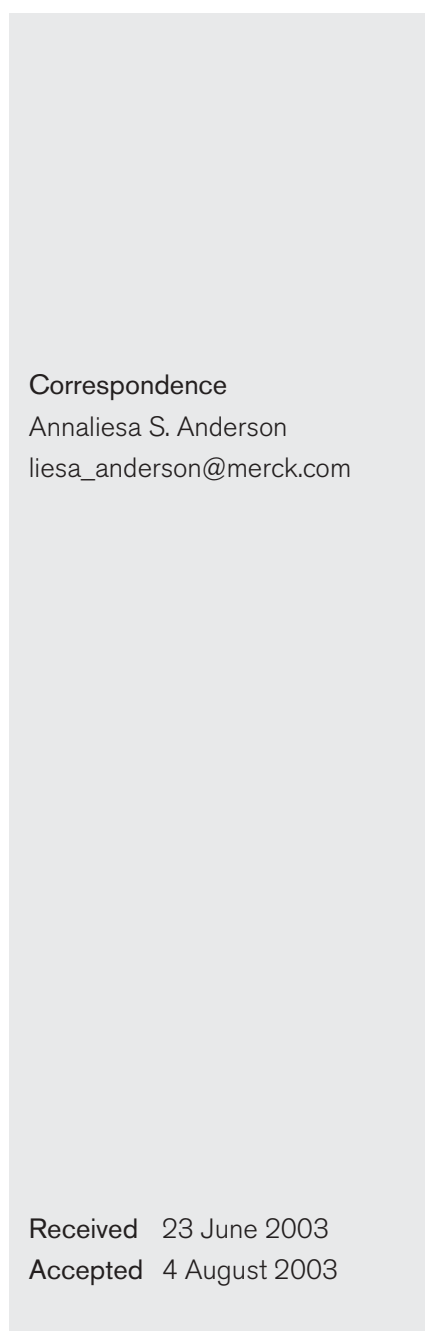

\title{
Evaluation of a multilocus sequence typing system for Staphylococcus epidermidis
}

\author{
Xin-Min Wang, ${ }^{1}$ Liliane Noble, $^{2}$ Barry N. Kreiswirth, ${ }^{3}$ \\ William Eisner, ${ }^{3}$ William McClements, ${ }^{1}$ Kathrin U. Jansen ${ }^{1}$ \\ and Annaliesa S. Anderson ${ }^{2}$ \\ ${ }^{1}$ Merck and Co. Inc., PO Box 4 WP 26-265, Sumneytown Pike, West Point, PA 19486, USA \\ ${ }^{2}$ Merck and Co. Inc., PO Box 2000 RY80Y 230, 126 E. Lincoln Ave., Rahway, NJ 07065, USA \\ ${ }^{3}$ PHRI TB Center, Public Health Research Institute, 225 Warren Street, Newark, NJ 07103, USA
}

\begin{abstract}
Staphylococcus epidermidis is a significant cause of nosocomial disease. However, the taxonomy of this pathogen, particularly at subspecies level, is unclear. A multilocus sequence typing (MLST) scheme has therefore been investigated as a tool to elucidate taxonomic relationships within this group, based on genetic relatedness. DNA sequences for internal fragments of seven housekeeping genes were compared in $\mathbf{4 7}$ geographically and temporally diverse $S$. epidermidis isolates that were obtained from clinical infections. Twenty-three different allelic profiles were detected; 17 of these were represented by single strains and the largest profile group contained 17 isolates. Diversity of the same collection of isolates was investigated by using PFGE of Smal-digested genomic DNA to test the discrimination and validity of the MLST approach. Isolates within the largest profile group were resolved into four distinct PFGE clusters on the basis of their Smal digest patterns. Isolates within other profile groups that contained multiple isolates had matching PFGE Smal patterns within each group. It appears that MLST is an effective method for grouping $S$. epidermidis strains at the subspecies level; however, it is not as discriminatory as it has been for other species for which MLST schemes have been established and, used alone, would not be a useful method for epidemiological studies. In addition, it was demonstrated that this method was effective for confirming the identity of S. epidermidis CoNS (coagulase-negative) isolates.
\end{abstract}

\section{INTRODUCTION}

The genus Staphylococcus is a member of the family Micrococcaceae and consists of over 40 species and subspecies at the time of writing. Staphylococci are differentiated by their ability to produce coagulase into the coagulase-positive (Staphylococcus aureus) and coagulase-negative (CoNS) groups (Lina et al., 2000). Within the CoNS group, Staphylococcus epidermidis is the species that is associated most commonly with disease. The Surveillance and Control of Pathogens of Epidemiologic Importance (SCOPE) study reported that of 5000 bloodstream infections from 41 US hospitals that were assessed during the 1995-1996 reporting

Abbreviations: CoNS, coagulase-negative staphylococci; MLST, multilocus sequence typing; NICU, neonatal intensive-care unit; RAPD, randomly amplified polymorphic DNA; ST, sequence type.

The GenBank/EMBL/DDBJ accession numbers for the sequences generated by this work are as follows: yqil1 -yqil10, AY163262AY163271; tpi1-tpi9, AY163272-AY163280; pta1-pta6, AY163281-Y163286; gmk1-gmk7, AY163287-AY163293; glpF1glpF9, AY163294-AY163302; aroE1-aroE10, AY163303AY163312; hsp1-hsp7, AY323453-AY323459. period, CoNS were the major cause (30.5\%); when 787 of these isolates were speciated, 602 (78\%) were identified as S. epidermidis (Marshall et al., 1998). Despite the clinical relevance of this organism, relatively little is known about its taxonomy at the subspecies level. This is partly because once a staphylococcal isolate has been assigned to the CoNS group, it is often not identified to the species level. Understanding of the diversity within and relationships among pathogenic taxa is an important prerequisite to meaningful taxonomic classification, accurate identification and pathogen detection and for epidemiology studies. Several taxonomic studies of $S$. epidermidis have been undertaken by using molecular techniques; these include PFGE (Linhardt et al., 1992; Bannerman et al., 1997); DNADNA hybridization (Villari et al., 2000); repetitive element sequence-based PCR (Hu \& Totake, 1997); sequence analysis of pathogenicity islands (Nahvi et al., 2001) and specific genes (Deplano et al., 1997); randomly amplified polymorphic DNA (RAPD) (Bogado et al., 2002; Dautle et al., 2002); PCR-amplified fragment length polymorphism (AFLP) (Sloos et al., 1998); ribotyping (Geary et al., 1997); plasmid profiles and phenotypic techniques, including anti- 
biograms (Sloos et al., 1998); and API identification strips (Sloos et al., 2000). However, these studies are often laboratory-specific and not polyphasic, making it difficult to correlate results and to assess true diversity within this clinically important bacterial species.

PFGE is the favoured method for S. epidermidis epidemiological studies and is useful for tracking specific outbreaks. Its utility is based on direct comparison of RFLPs of the entire genome when restricted with a rare-cutting enzyme. Given that mutations can occur at a relatively high frequency, including in the rare-cutting restriction sites, a single genetic alteration can alter three bands in a PFGE pattern (Tenover et al., 1995). Increased changes over time limit the use of PFGE analysis for taxonomic studies and in long-term or multicentre studies. This method also has the drawback of being extremely time-consuming and subjective in interpretation and it is difficult to record PFGE patterns and their identification. As with the other molecular methods listed above, PFGE does not transfer well between laboratories, as demonstrated by van Belkum et al. (1998), who conducted a large interlaboratory study testing the reproducibility of PFGE for staphylococci. Although the method is robust, it is subject to operator variation.

An alternative molecular classification method is multilocus sequence typing (MLST), a sequence-based method first described by Maiden et al. (1998), where it was used to delimit strain associations and clonal groupings within a reference set of Neisseria meningitidis isolates. It was developed as a robust typing method that measures sequence divergence of several genes that are subject to slow rates of change, thus making it suitable for long-term epidemiological studies. Furthermore, as data are in the form of sequences, they can be catalogued easily and compared readily among laboratories. This method has since been demonstrated to be an effective method for the study of taxonomic and genetic diversity of other microbial pathogens, including Streptococcus pneumoniae (Enright \& Spratt, 1998), Staphylococcus aureus (Enright et al., 2000), Campylobacter jejuni (Dingle et al., 2001), Salmonella spp. (Kotetishvili et al., 2002), Candida albicans (Bougnoux et al., 2002) and Enterococcus faecalis (Nallapareddy et al., 2002).

The aim of this study was to evaluate the use of an MLSTbased scheme to study the diversity of a set of S. epidermidis isolates selected randomly from a collection of isolates that were obtained from geographically dispersed hospitals and associated with different disease states.

\section{METHODS}

Bacterial strains and culture conditions. Bacterial strains investigated in this study are listed in Table 1. S. epidermidis strains were of clinical origin and were isolated from various sites and geographical areas. Strains were initially received on tryptone soy agar (TSA) II+5 \% sheep blood agar (BBL; Becton Dickinson) and transferred subsequently to Microbank porous carrier beads (Pro-Lab Diagnostics) for long-term storage at $-80{ }^{\circ} \mathrm{C}$. Cultures were grown by inoculating TSA II $+5 \%$ sheep blood agar plates directly with a carrier bead and incubating at $37^{\circ} \mathrm{C}$ overnight. A clonal isolate was picked to inoculate $3 \mathrm{ml}$ tryptic soy broth (Difco) and incubated overnight at $37^{\circ} \mathrm{C}$ with shaking at 150 r.p.m.

Small-scale isolation of chromosomal DNA. Overnight cultures were centrifuged in $1.5 \mathrm{ml}$ Eppendorf tubes at 14000 r.p.m. for $5 \mathrm{~min}$. Broth was decanted and pellets were resuspended in $500 \mu \mathrm{l}$ resuspension buffer ( $25 \%$ sucrose, $10 \mathrm{mM}$ Tris, $\mathrm{pH} 7 \cdot 5$ ). Suspensions were lysed with $20 \mu \mathrm{g}$ lyphostatin $\mathrm{ml}^{-1}$ (Sigma-Aldrich) and were incubated at $37^{\circ} \mathrm{C}$ for $1 \mathrm{~h}$. At the end of the incubation period, $250 \mu \mathrm{l} 2 \%$ SDS was added to each tube and vortexed until the viscosity of the solution decreased noticeably. Phenol/chloroform/isoamyl alcohol solution $(250 \mu \mathrm{l})$ $(25: 24: 1, \mathrm{v} / \mathrm{v})$ (Gibco/Invitrogen) was added. The mixture was vortexed for $30 \mathrm{~s}$ and centrifuged for $5 \mathrm{~min}$ at 14000 r.p.m. The aqueous phase was removed and the precipitation steps were repeated until almost no interface remained; $0 \cdot 1$ vols $3 \mathrm{M} \mathrm{NaOAc}, \mathrm{pH} 4 \cdot 8$, were added to each tube and mixed. One volume of 2-propanol was then added and the tube was mixed again. Tubes were left to incubate for $5 \mathrm{~min}$ at room temperature and then centrifuged at 14000 r.p.m. for $15 \mathrm{~min}$. Supernatant was decanted and tubes were allowed to dry upside-down on tissue. Pellets were resuspended in $50 \mu \mathrm{l}$ sterile $\mathrm{H}_{2} \mathrm{O}$. Multiple $(5 \times)$ DNA preparations were performed on one isolate $(S$. epidermidis ATCC 35984) to evaluate the robustness of the technique.

MLST. Nucleotide sequences of seven S. epidermidis housekeeping genes, shikimate dehydrogenase, heat-shock protein, glycerol kinase, guanylate kinase, phosphate acetyltransferase, triphosphate isomerase and acetyl coenzyme A acetyltransferase, were obtained from GenBank and PCR primers were designed to amplify fragments that ranged from 491 to $620 \mathrm{bp}$ in size (Table 2). Approximately $50 \mathrm{ng}$ template bacterial DNA was PCR-amplified with each primer set by using a BD Advantage cDNA PCR kit (BD Clontech) according to the manufacturer's instructions and an Applied Biosystems GeneAmp 9700 thermocycler. An initial denaturation at $94^{\circ} \mathrm{C}$ for 1 min was followed by 30 cycles of denaturation at $94{ }^{\circ} \mathrm{C}$ for $1 \mathrm{~min}$, annealing at $53{ }^{\circ} \mathrm{C}$ for $30 \mathrm{~s}$ and extension at $68{ }^{\circ} \mathrm{C}$ for $4 \mathrm{~min}$ and a final extension at $68{ }^{\circ} \mathrm{C}$ for $4 \mathrm{~min}$. Amplified DNA was purified with a QIAquick PCR Purification kit (Qiagen) and both strands were sequenced by using BigDye fluorescent terminators (Applied Biosystems) and the amplification primers on an ABI 377 DNA Sequencer ( $36 \mathrm{~cm}$ gel for $7 \mathrm{~h}$ ). Sequences were analysed by using Sequencer 3.1 (GeneCodes).

Sequences obtained were assigned allele numbers that were used to generate sequence types (STs) for each isolate. Allelic profiles were compared by using the simple matching coefficient and clustered by UPGMA (NTSYSpc; Exeter Publishing). BURST analysis was done by using software from the MLST site (http://www.mlst.net).

PFGE. Each isolate was genotyped by PFGE using the restriction enzyme SmaI (New England BioLabs) and performed according to methods described previously (Roberts et al., 1998). Macrorestriction profiles were interpreted as described by Tenover et al. (1995).

Resistance to methicillin. MIC values of the isolates to methicillin were determined at their hospitals of origin by using standard procedures. Bacterial suspensions in water were adjusted to a McFarland standard of $0 \cdot 5$. In vitro susceptibility tests (MIC) were conducted by inoculating microwell susceptibility test cards; analysis was performed by the bioMérieux Vitek automated system. MIC values were determined according to National Committee for Clinical Laboratory Standards (NCCLS) guidelines. Isolates with MIC values to oxacillin that were below $2 \mathrm{mg} \mathrm{l}^{-1}$ were considered to be susceptible to methicillin and those with MIC values above $2 \mathrm{mg} \mathrm{l}^{-1}$ were considered to be resistant. To confirm these results, chromosomal digests were probed for the presence of the mecA gene by using the method described by Kreiswirth et al. (1993). 
Table 1. Staphylococci used in this investigation and corresponding STs, BURST groupings and PFGE profiles

ATCC, American Type Culture Collection; MB, Merck bacteria collection; MCL, Merck clinical collection; MRSA, methicillin-resistant Staphylococcus aureus; R, resistant; S, susceptible.

\begin{tabular}{|c|c|c|c|c|c|c|c|c|c|c|c|c|c|}
\hline \multirow[t]{2}{*}{ Organism (strain) } & \multirow[t]{2}{*}{ Source } & \multicolumn{7}{|c|}{ Allelic profile } & \multirow{2}{*}{$\begin{array}{c}\text { ST } \\
\text { group }\end{array}$} & \multirow{2}{*}{$\begin{array}{l}\text { BURST } \\
\text { group }\end{array}$} & \multirow{2}{*}{$\begin{array}{l}\text { PFGE } \\
\text { profile }\end{array}$} & \multirow{2}{*}{$\begin{array}{l}\text { Methicillin } \\
\text { resistance }\end{array}$} & \multirow{2}{*}{$\begin{array}{l}\operatorname{mec} A \\
\text { gene }\end{array}$} \\
\hline & & aroE & $g l p F$ & $g m k$ & pta & $t p i$ & $y q i L$ & $h s p$ & & & & & \\
\hline \multicolumn{14}{|c|}{ Staphylococcus epidermidis: } \\
\hline RP62A & $\begin{array}{c}\text { Also known as ATCC 35984; catheter } \\
\text { sepsis, Tennessee, USA (pre-1982) }\end{array}$ & 1 & 1 & 1 & 1 & 1 & 1 & 1 & 1 & $S$ & & S & + \\
\hline MB2230 & Also known as ATCC 12228 & 1 & 2 & 2 & 2 & 1 & 1 & 1 & 2 & 1 & $2-2$ & s & - \\
\hline 1 & Urine, Lexington, KY, USA; MCL8616 & 1 & 2 & 2 & 2 & 1 & 1 & 1 & 2 & 1 & $2-1$ & $\mathrm{R}$ & + \\
\hline 2 & $\begin{array}{l}\text { Exudate right inquina, Sun City, AZ, USA; } \\
\text { MCL8719 }\end{array}$ & 1 & 2 & 2 & 2 & 1 & 1 & 1 & 2 & 1 & $2-1$ & $\mathrm{R}$ & + \\
\hline 3 & $\begin{array}{l}\text { Left shoulder, Ft Myers, FL, USA; } \\
\text { MCL } 8758\end{array}$ & 1 & 2 & 2 & 2 & 1 & 1 & 1 & 2 & 1 & $2-1$ & $\mathrm{R}$ & + \\
\hline 4 & Pus, Kalafong, South Africa; MCL12366 & 1 & 2 & 2 & 2 & 1 & 1 & 1 & 2 & 1 & $2-1$ & $\mathrm{R}$ & + \\
\hline 5 & $\begin{array}{l}\text { Catheter, uterus, Pretoria, South Africa; } \\
\text { MCL12370 }\end{array}$ & 1 & 2 & 2 & 2 & 1 & 1 & 1 & 2 & 1 & $2-1$ & s & + \\
\hline 6 & $\begin{array}{l}\text { Exudate, abdomen, Philadelphia, PA, USA; } \\
\text { MCL12546 }\end{array}$ & 1 & 2 & 2 & 2 & 1 & 1 & 1 & 2 & 1 & $2-1$ & s & - \\
\hline 7 & Peritoneum, Buffalo, NY, USA; MCL8225 & 1 & 2 & 2 & 2 & 1 & 1 & 1 & 2 & 1 & $2-2$ & $\mathrm{R}$ & + \\
\hline 8 & Left ankle, Springfield, IL, USA; MCL8847 & 1 & 2 & 2 & 2 & 1 & 1 & 1 & 2 & 1 & $2-2$ & $\mathrm{R}$ & + \\
\hline 9 & $\begin{array}{l}\text { Pus, appendix, Parow, South Africa; } \\
\text { MCL12296 }\end{array}$ & 1 & 2 & 2 & 2 & 1 & 1 & 1 & 2 & 1 & $2-2$ & $\mathrm{R}$ & + \\
\hline 10 & $\begin{array}{l}\text { Abdominal wound, Torrance, CA, USA; } \\
\text { MCL } 8888\end{array}$ & 1 & 2 & 2 & 2 & 1 & 1 & 1 & 2 & 1 & $2-3$ & $\mathrm{R}$ & + \\
\hline 11 & Urine, Kent, WA, USA; MCL8519 & 1 & 2 & 2 & 2 & 1 & 1 & 1 & 2 & 1 & $2-3$ & s & - \\
\hline 12 & Blood, Los Angeles, CA, USA; MCL8321 & 1 & 2 & 2 & 2 & 1 & 1 & 1 & 2 & 1 & $2-4$ & $\mathrm{R}$ & + \\
\hline 13 & Right foot, Wichita, KS, USA; MCL8568 & 1 & 2 & 2 & 2 & 1 & 1 & 1 & 2 & 1 & $2-4$ & $\mathrm{R}$ & + \\
\hline 14 & $\begin{array}{l}\text { Left foot, New Orleans, LA, USA; } \\
\text { MCL } 8751\end{array}$ & 1 & 2 & 2 & 2 & 1 & 1 & 1 & 2 & 1 & $2-4$ & $\mathrm{R}$ & + \\
\hline 15 & Blood, Providence, RI, USA; MCL10478 & 1 & 2 & 2 & 2 & 1 & 1 & 1 & 2 & 1 & $2-4$ & s & + \\
\hline 16 & $\begin{array}{l}\text { Right arm pus, Baltimore, MD, USA; } \\
\text { MCL8952 }\end{array}$ & 1 & 2 & 2 & 2 & 1 & 1 & 1 & 2 & 1 & & $\mathrm{R}$ & + \\
\hline 17 & $\begin{array}{l}\text { Peritoneum, Los Angeles, CA, USA; } \\
\text { MCL8192 }\end{array}$ & 1 & 2 & 6 & 2 & 1 & 1 & 1 & 3 & 1 & & $\mathrm{R}$ & + \\
\hline 18 & $\begin{array}{l}\text { Intra-abdominal, NYC, NY, USA; } \\
\text { MCL8359 }\end{array}$ & 1 & 2 & 6 & 2 & 1 & 1 & 1 & 3 & 1 & & $\mathrm{R}$ & + \\
\hline 19 & Pus, Pretoria, South Africa; MCL11771 & 1 & 2 & 6 & 2 & 1 & 1 & 1 & 3 & 1 & & s & + \\
\hline 20 & $\begin{array}{l}\text { Endometrium, Los Angeles, CA, USA; } \\
\text { MCL12846 }\end{array}$ & 1 & 2 & 6 & 2 & 1 & 1 & 1 & 3 & 1 & & s & - \\
\hline
\end{tabular}




\begin{tabular}{|c|c|c|c|c|c|c|c|c|c|c|c|c|c|}
\hline \multirow[t]{2}{*}{ Organism (strain) } & \multirow[t]{2}{*}{ Source } & \multicolumn{7}{|c|}{ Allelic profile } & \multirow{2}{*}{$\begin{array}{c}\text { ST } \\
\text { group }\end{array}$} & \multirow{2}{*}{$\begin{array}{l}\text { BURST } \\
\text { group }\end{array}$} & \multirow{2}{*}{$\begin{array}{l}\text { PFGE } \\
\text { profile }\end{array}$} & \multirow{2}{*}{$\begin{array}{l}\text { Methicillin } \\
\text { resistance }\end{array}$} & \multirow{2}{*}{$\begin{array}{l}\operatorname{mec} A \\
\text { gene }\end{array}$} \\
\hline & & aroE & $g l p F$ & gmk & $p t a$ & $t p i$ & $y q i L$ & hsp & & & & & \\
\hline 21 & Blood, NYC, NY, USA; MCL11615 & 1 & 2 & 2 & 2 & 7 & 1 & 1 & 4 & 1 & & $\mathrm{R}$ & + \\
\hline 22 & Pus, wound, South Africa; MCL12307 & 1 & 2 & 2 & 2 & 7 & 1 & 1 & 4 & 1 & & $\mathrm{R}$ & + \\
\hline 23 & Exudate, face, Brazil; MCL12000 & 2 & 3 & 3 & 3 & 2 & 2 & 2 & 5 & S & & $\mathrm{R}$ & - \\
\hline 24 & Right arm, Springfield, IL, USA; MCL8827 & 2 & 3 & 3 & 3 & 2 & 2 & 2 & 5 & S & & s & - \\
\hline 25 & Abdomen, Springfield, IL, USA; MCL8851 & 3 & 2 & 2 & 2 & 1 & 1 & 1 & 6 & 1 & & $\mathrm{R}$ & + \\
\hline 26 & $\begin{array}{l}\text { Incision, wound, Wilmington, DE, USA; } \\
\text { MCL9430 }\end{array}$ & 1 & 2 & 2 & 1 & 1 & 1 & 1 & 7 & 1 & & $\mathrm{R}$ & + \\
\hline 27 & Left arm, Sun City, AZ, USA; MCL9689 & 1 & 2 & 2 & 1 & 1 & 1 & 1 & 7 & 1 & & $\mathrm{R}$ & + \\
\hline 28 & Wound, Orange, CA, USA; MCL10141 & 1 & 2 & 2 & 1 & 1 & 1 & 1 & 7 & 1 & & S & + \\
\hline 29 & $\begin{array}{l}\text { Intra-peritoneal pus, Buffalo, NY, USA; } \\
\text { MCL9891 }\end{array}$ & 1 & 2 & 2 & 2 & 1 & 3 & 1 & 8 & 1 & & $\mathrm{R}$ & + \\
\hline 30 & $\begin{array}{l}\text { Right tibial wound, Honolulu, HA, USA; } \\
\text { MCL8453 }\end{array}$ & 1 & 2 & 2 & 2 & 1 & 3 & 1 & 8 & 1 & & $\mathrm{R}$ & + \\
\hline 31 & $\begin{array}{l}\text { Right leg tissue, Wichita, KS, USA; } \\
\text { MCL8809 }\end{array}$ & 1 & 2 & 1 & 1 & 3 & 1 & 1 & 9 & $S$ & & S & - \\
\hline 32 & $\begin{array}{l}\text { Pus, gall bladder, Wilmington, DE, USA; } \\
\text { MCL9423 }\end{array}$ & 1 & 2 & 2 & 1 & 1 & 10 & 1 & 10 & 1 & & $\mathrm{R}$ & + \\
\hline 33 & Blood, Miraflores, Peru; MCL10484 & 9 & 4 & 1 & 1 & 5 & 4 & 4 & 11 & S & & s & + \\
\hline 34 & Blood, Pretoria, South Africa; MCL12281 & 1 & 2 & 2 & 6 & 1 & 1 & 1 & 12 & 1 & & $\mathrm{R}$ & + \\
\hline 35 & Pus, right leg, Lima, Peru; MCL11238 & 10 & 5 & 1 & 4 & 8 & 8 & 7 & 13 & S & & $\mathrm{R}$ & - \\
\hline 36 & $\begin{array}{l}\text { Post-operative, back, Tacoma, WA, USA; } \\
\text { MCL8040 }\end{array}$ & 1 & 2 & 5 & 2 & 1 & 8 & 3 & 14 & S & & $\mathrm{R}$ & + \\
\hline 37 & Blood, Mexico City, Mexico; MCL11657 & 1 & 2 & 6 & 2 & 1 & 7 & 1 & 15 & 1 & & $\mathrm{R}$ & + \\
\hline 38 & $\begin{array}{l}\text { Exudate right inquina, Sun City, AZ, USA; } \\
\text { MCL8778 }\end{array}$ & 1 & 8 & 2 & 2 & 1 & 1 & 1 & 16 & 1 & & s & + \\
\hline 39 & $\begin{array}{l}\text { Vaginal swab, Kalafong Hospital, South } \\
\text { Africa; MCL13231 }\end{array}$ & 1 & 9 & 4 & 3 & 6 & 9 & 2 & 17 & S & & s & - \\
\hline 40 & Left leg, Springfield, IL, USA; MCL8834 & 4 & 5 & 1 & 2 & 4 & 4 & 5 & 18 & S & & $\mathrm{R}$ & - \\
\hline 41 & $\begin{array}{l}\text { Peritoneal fluid, Somers Pt, NJ, USA; } \\
\text { MCL8566 }\end{array}$ & 5 & 4 & 1 & 2 & 1 & 8 & 4 & 19 & S & & $\mathrm{R}$ & - \\
\hline 42 & Skin left leg, Ft Myers, FL, USA; MCL8544 & 6 & 6 & 7 & 5 & 4 & 5 & 5 & 20 & $S$ & & s & - \\
\hline 43 & $\begin{array}{l}\text { Right leg wound, Baltimore, MD, USA; } \\
\text { MCL8798 }\end{array}$ & 7 & 7 & 2 & 6 & 1 & 6 & 6 & 21 & $S$ & & $\mathrm{R}$ & - \\
\hline 44 & Appendix pus, Brazil; MCL10233 & 8 & 6 & 7 & 1 & 9 & 11 & 5 & 22 & S & & $\mathrm{R}$ & - \\
\hline 45 & $\begin{array}{l}\text { Endometrium, Los Angeles, CA, USA; } \\
\text { MCL10147 }\end{array}$ & 1 & 2 & 2 & 2 & 1 & 1 & 3 & 23 & 1 & & s & + \\
\hline Staphylococcus xylosus ATCC 35033 & ATCC strain & - & - & - & - & - & - & & - & - & - & & \\
\hline
\end{tabular}




\begin{tabular}{|c|c|c|c|c|c|c|c|c|c|c|}
\hline $\begin{array}{l}\text { Staphylococcus saprophyticus MB } 4742 \\
\text { Staphylococcus capitis: }\end{array}$ & NRRL B2628 & - & - & - & - & - & - & - & - & - \\
\hline MB5230 & ATCC $27840^{\mathrm{T}}$ & - & - & - & - & - & - & - & - & - \\
\hline MB5347 & ATCC 27843 & - & - & - & - & - & - & - & - & - \\
\hline Staphylococcus hominis MB5231 & ATCC $27844^{\mathrm{T}}$ & - & - & - & - & - & - & - & - & - \\
\hline Staphylococcus sciuri MB5349 & ATCC 29060 & - & - & - & - & - & - & - & - & - \\
\hline Staphylococcus haemolyticus MB5411 & Wound, Grand Forks, ND, USA & - & - & - & - & - & - & - & - & - \\
\hline Staphylococcus aureus COL & MRSA strain & - & - & - & - & - & - & - & - & - \\
\hline
\end{tabular}

Table 2. Genes and PCR primers used for S. epidermidis MLST

\begin{tabular}{|c|c|c|c|c|c|}
\hline Gene & GenBank no. & PCR primers & $\begin{array}{l}\text { Amplified } \\
\text { fragment size } \\
\text { (bp) }\end{array}$ & $\begin{array}{c}\text { Allele } \\
\text { region } \\
\text { (bp) }\end{array}$ & $\begin{array}{l}\text { No. } \\
\text { alleles }\end{array}$ \\
\hline Shikimate dehydrogenase (aroE) & AF269525 & Forward, TCTCATTCATTATCGCCATTG; reverse, CTAAAGTATGTGAAGCTAAACG & 586 & 482 & 10 \\
\hline Guanylate kinase (gmk) & AF270133 & Forward, CTGTTAATTGTTCTTTCAGGCC; reverse, ATTTAGCTTCGATTCGCTCTC & 574 & 469 & 7 \\
\hline Phosphate acetyltransferase (pta) & AF269661 & Forward, TTAAGGAGGATATTATGGCTG; reverse, GCTTAACATAGCTACTTTAGG & 620 & 499 & 6 \\
\hline Triphosphate isomerase $(t p i)$ & AF269838 & Forward, CAATCCAATTAGACGCTTTAG; reverse, TAATGATGCGCCACCTACAAG & 593 & 424 & 9 \\
\hline Acetyl coenzyme A acetyltransferase (yqil) & AF270323 & Forward, CACGCATAGTATTAGCTGAAG; reverse, GTTGTGGTACCTCTACAGGTAC & 615 & 473 & 11 \\
\hline
\end{tabular}




\section{RESULTS AND DISCUSSION}

BLAST searches were done in GenBank with the seven MLST alleles used for typing $S$. aureus, as described by Enright et al. (2000). Sequence equivalents were found in S. epidermidis for all of the alleles except for the carbamate kinase ( $\operatorname{arcC})$ allele. Heat-shock protein (Hsp) was included in the study to ensure that the analysis was done with the appropriate number of alleles (seven). PCR primers (Table 2) were successful in amplifying PCR products of the expected length from all S. epidermidis strains tested. The same PCR primers were used to screen a strain of $S$. aureus and seven non-S. epidermidis CoNS strains, to test the specificity of the PCR primers for S. epidermidis strains. No PCR products were obtained for the $S$. aureus strain or for the majority of CoNS strains (Table 3). Where PCR products were obtained, they were not of the expected size. Sequence analysis of these products revealed that they were not the target sequences.

PCR products obtained from the S. epidermidis allelic screen were all sequenced (both strands) and analysed for allelic variation. The number of different variants for each locus ranged from six to ten. The most variable locus was aroE, which had a total of 28 polymorphic sites; tpi was the least variable with 12 polymorphic sites. The allelic profile obtained for each S. epidermidis strain is given in Table 1. In total, 23 STs were identified for the 47 isolates (Table 1). The largest ST group (group 2) contained 17 isolates; there were five additional groups that contained more than one strain and the remaining 17 ST groups contained single isolates. STs were compared by cluster and BURST analyses. For BURST analysis, a clonal group was considered to be strains with an ST that shared at least six alleles. One clonal group that contained 11 STs (representing 34 isolates) was detected. All other STs were non-clonal ('singletons'). BURST groupings were confirmed by cluster analysis, which showed that isolates within this group displayed $76 \%$ similarity (Fig. 1). The most divergent STs were 5, 17, 20 and 22, which all fell away from the main cluster with no shared alleles.

PFGE with SmaI-digested DNA was performed on the isolates to determine how the two methods compared. The largest ST group (group 2) contained strains with SmaI restriction profiles that fell into four groups (Fig. 2a). The remaining ST groups that contained more than one isolate had similar profiles within their ST groups, with differences that ranged from none to two bands (Fig. 2b). Cluster analysis of all SmaI restriction profiles demonstrated the similarity of the isolates within the ST groups to which they were assigned, with just two groups ( 2 and 3 ) clustering away from the corresponding group members (Fig. 2c). Two main clusters were resolved, one of which contained six 'singleton' BURST clonal groups and one that contained a mixture of 'singleton' BURST clonal groups and BURST clonal group 1 isolates. The clustering pattern was similar to that obtained for the MLST profiles (Fig. 1).

Methicillin-resistance data were provided for all isolates in the collection; 31 (67\%) were reported to be resistant (Table $1)$. To confirm these results, isolates were also probed for the presence of the mecA gene by Southern hybridization; by using this method, 33 (70\%) contained the gene. Inconsistencies with MIC values were attributed to these being determined in several different laboratories. Strains were deemed to be resistant if they contained the mecA gene, in accordance with NCCLS guidelines. The resistance status of each strain is also marked on the MLST and PFGE dendrograms (Figs 1 and 2c). The methicillin-resistance genotype appeared to be randomly distributed among strains, suggesting that it has been acquired randomly. The largest ST group (group 2) contained $14 \mathrm{mec} A$-positive strains and three that were negative; the gene was not confined to specific PFGE profiles within this group. It was also observed that isolates

Table 3. Specificity of MLST primers for S. epidermidis

S, PCR product size is similar to that of S. epidermidis by gel electrophoresis; however, sequence is not S. epidermidis-like (21-27\% mismatch in pta and $15-18 \%$ mismatch in $h s p)$; N, PCR product sizes were different from that of $S$. epidermidis; -, no amplification was observed.

\begin{tabular}{|lccccccc|}
\hline Species & \multicolumn{7}{c|}{ Locus } \\
\cline { 2 - 8 } & $\boldsymbol{a r o \boldsymbol { E }}$ & $\boldsymbol{g l p \boldsymbol { F }}$ & $\boldsymbol{g m \boldsymbol { k }}$ & $\boldsymbol{p} \boldsymbol{t a}$ & $\boldsymbol{t} \boldsymbol{p i}$ & $\boldsymbol{y} \boldsymbol{q} \boldsymbol{i L}$ & $\boldsymbol{h} \boldsymbol{p}$ \\
\hline S. epidermidis & $586^{*}$ & 616 & 574 & 620 & 593 & 615 & 491 \\
S. xylosus & $\mathrm{N}$ & - & $\mathrm{N}$ & $\mathrm{N}$ & $\mathrm{N}$ & $\mathrm{N}$ & - \\
S. saprophyticus & $\mathrm{N}$ & - & $\mathrm{N}$ & $\mathrm{S}$ & $\mathrm{N}$ & - & $\mathrm{S}$ \\
S. capitis & $\mathrm{N}$ & - & $\mathrm{N}$ & $\mathrm{S}$ & $\mathrm{N}$ & - & $\mathrm{S}$ \\
S. hominis & $\mathrm{N}$ & - & $\mathrm{N}$ & $\mathrm{N}$ & $\mathrm{N}$ & - & $\mathrm{S}$ \\
S. sciuri & $\mathrm{N}$ & - & $\mathrm{N}$ & $\mathrm{N}$ & $\mathrm{N}$ & $\mathrm{N}$ & $\mathrm{N}$ \\
S. haemolyticus & $\mathrm{N}$ & - & $\mathrm{N}$ & $\mathrm{N}$ & $\mathrm{N}$ & - & $\mathrm{N}$ \\
S. aureus COL & $\mathrm{N}$ & - & - & $\mathrm{N}$ & $\mathrm{N}$ & - & $\mathrm{S}$ \\
\hline
\end{tabular}

${ }^{*}$ Size of sequence $(\mathrm{bp})$. 


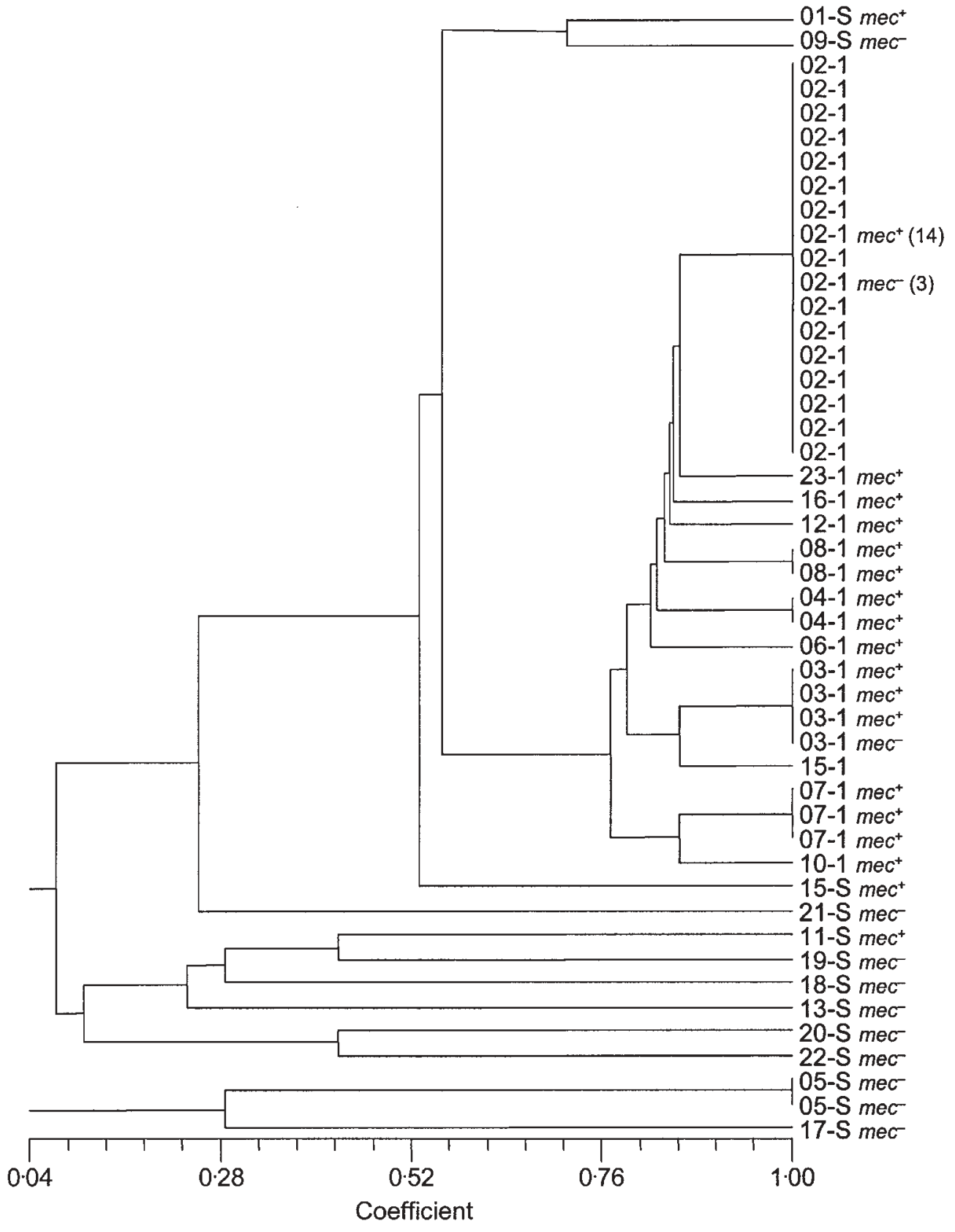

Fig. 1. Dendrogram showing genetic relatedness of 47 S. epidermidis isolates. Data were compared by using the simple matching coefficient and clustered by UPGMA.

from the singleton group were predominantly mecA-negative; however, as they were not represented by multiple isolates, this observation may not be significant.

S. epidermidis isolates used in this study originated from a diverse range of infections and geographical locations and were cultured in the years 1996-1999. Thirty-three isolates were obtained from continental USA, one from Hawaii, one from Europe, five from South America and seven from South Africa. In total, 23 different STs were found, five of which contained more than one isolate. No correlation was observed between an isolate's ST and its infection site or geographical location. The largest group (group 2) was composed of strains from continental USA, Europe and South Africa. STs 3 and 4 contained isolates from South Africa and continental USA; ST 5 contained isolates from Brazil and continental USA and isolates from ST 8 were from Hawaii and continental USA. Within BURST clonal group 1, there was also a global distribution of strain types, with isolates from the USA, Europe, South Africa and Mexico. 


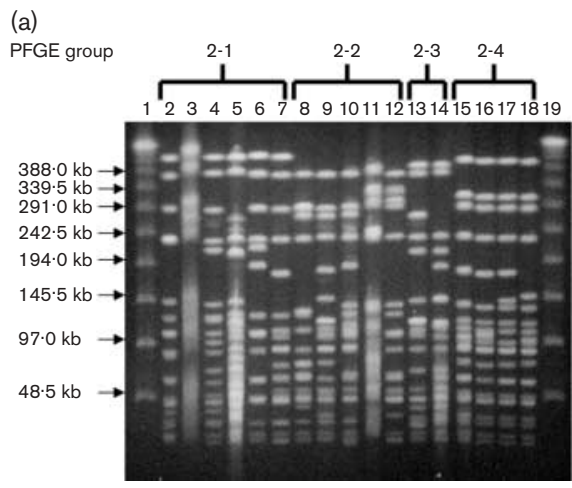

(b)

MLST group
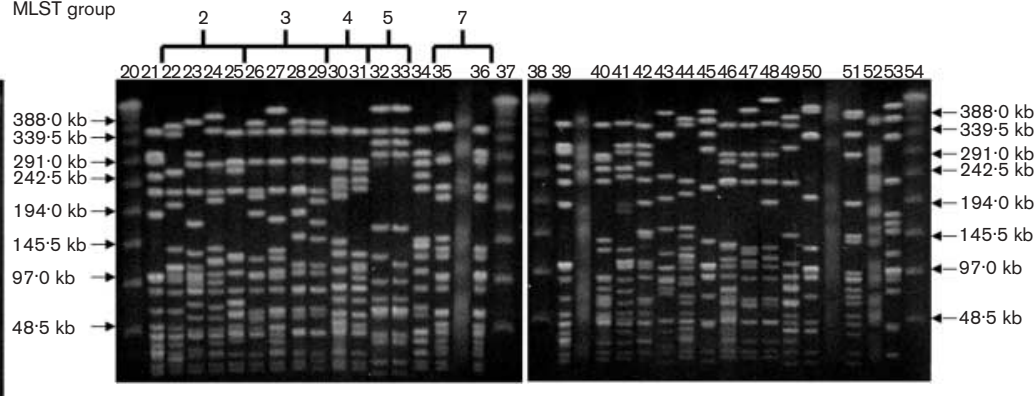

(c)

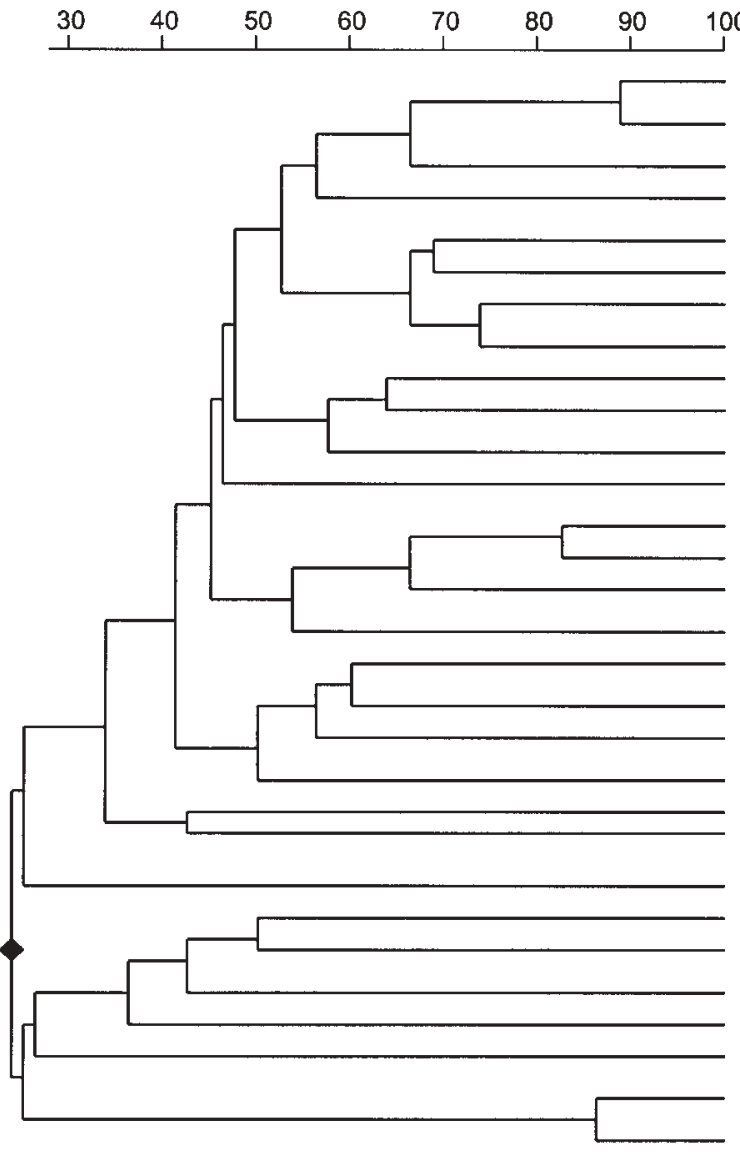

ID

ST-BURSTGp mec gene)

Se 27

Se 28

(07-1+)

(07-1+)

Se 20

(03-1-)

Se 25

(06-1 +)

Se strains ST 2-4 (02-1 +)

Se 21

Se 22

$(04-1+)$

(04-1 +)

Se 36

Se 38

$(14-S+)$

$(16-1+)$

Se 37

$(15-1+)$

Se 29

$(08-1+)$

$(12-1+)$

(03-1 +)

Se 17

(03-1+)

Se 18

$(03-1+)$

Se 19

(03-1+/-)

Se 32

Se RP62A

$(10-1+)$

$(01-S+)$

$(09-S+)$

(17-S -)

Se strains ST 2-1 $(02-1+/-)$

Se strains ST 2-2 $(02-1+/-)$

Se 43

$\mathrm{Se} 35$

Se 40

(21-S -)

(13-S -)

(18-S -)

(11-S +)

(20-S -)

Se 42

Se 44

(22-S -)

(05-S -)

Se 23

(05-S -)

Fig. 2. PFGE analysis of isolates by using Smal restriction digests. (a) Subgroupings within ST 2: lanes 1 and $19, \lambda$ ladder; lane $2, S$. epidermidis 1; lane 3, S. epidermidis 22; lane 4, S. epidermidis 3; lane 5, S. epidermidis 4; lane 6, S. epidermidis 6; lane 7, S. epidermidis 2; lane 8, S. epidermidis 7; lane 9, S. epidermidis 9; lane 10, S. epidermidis MB2230; lane 11, S. epidermidis 45; lane 12, S. epidermidis 8; lane 13, S. epidermidis 10; lane 14, S. epidermidis 11; lane 15, S. epidermidis 14; lane 16, S. epidermidis 12; lane 17, S. epidermidis 15; lane 18, S. epidermidis 13. (b) All PFGE profiles obtained, according to ST group: lanes 20, 37,38 and 54, $\lambda$ ladder; lane 21, RP62A; lane 22, S. epidermidis 10; lane 23, S. epidermidis 12; lane 24, S. epidermidis 3; lane 25, S. epidermidis 7; lane 26, S. epidermidis 19; lane 27, S. epidermidis 20; lane 28, S. epidermidis 17; lane 29, S. epidermidis 18; lane 30, S. epidermidis 21; lane 31, S. epidermidis 22; lane 32, S. epidermidis 23; lane 33, S. epidermidis 24; lane 34, S. epidermidis 25; lane 35, S. epidermidis 28; lane 36, S. epidermidis 27; lane 39, RP62A; lane 40, S. epidermidis 29; lane 41, S. epidermidis 31; lane 42, S. epidermidis 32; lane 43, S. epidermidis 33; lane 44, S. epidermidis 34; lane 45, S. epidermidis 35; lane 46, S. epidermidis 36; lane 47, S. epidermidis 37; lane 48, S. epidermidis 38; lane 49, S. epidermidis 39; lane 50, S. epidermidis 40; lane 51, S. epidermidis 42; lane 52, S. epidermidis 43; lane 53, S. epidermidis 44. ST groups that contain multiple isolates are marked. ST2 is represented by one strain from each of the PFGE subgroups. (c) Cluster analysis of Smal restriction profiles for S. epidermidis isolates. Analysis was performed by using the Jaquard coefficient and clustered by UPGMA. 
Isolates that did not correspond to a clonal group were derived from continental USA, Brazil, Peru and South Africa. As this was a pilot study (47 isolates, of which 33 were from continental USA), one cannot make generalized conclusions about the epidemiology of S. epidermidis disease. However, it does demonstrate that clonal types are distributed globally and that there does not appear to be a correlation between ST and diagnosed infection caused by the isolate. The clonal dissimilation of $S$. epidermidis strains is supported by the work of Miragaia et al. (2002), who used PFGE to type a large collection of isolates from Denmark $(n=136)$ and Iceland $(n=94)$ and detected 40 different clonal groups. Comparison of these groups with strains from Mexico $(n=74)$, Uruguay $(n=10)$, Greece $(n=34)$ and Cape Verde $(n=48)$ revealed that isolates had similar PFGE profiles that were independent of their geographical location.

To compare MLST with an established typing method, we also analysed the $S$. epidermidis isolates by PFGE. Twentythree patterns were observed by using the criteria proposed by Tenover et al. (1995). Taken alone, the PFGE data suggest a high degree of genotypic diversity within the S. epidermidis isolates studied. This mirrors other studies that have sought to type $S$. epidermidis strains by PFGE. Raimundo et al. (2002) typed 55 isolates from a neonatal intensive-care unit (NICU) and identified 43 subtypes by PFGE, compared to only 15 types by RAPD analysis. Sloos et al. (1998) typed 53 isolates from 24 neonates and found that 16 isolates (from six babies) had common PFGE profiles, whereas the remaining 37 isolates all gave unique profiles. In a second study, Sloos et al. (2000) characterized 16 epidemiologically distinct isolates by PFGE, all with unique profiles. We have demonstrated that MLST provides a more defined picture of taxonomic diversity, with $74.5 \%$ of strains originating from a single BURST clonal group and the remaining 13 types all being 'singletons'. The genetic diversity observed from the PFGE profiles suggests that $S$. epidermidis strains undergo a high degree of gene flux, more so than other species that have been typed by using MLST, as multiple PFGE types were not observed within single ST groups (Enright et al., 2000). It is to be expected that greater variation is observed by using PFGE than MLST, as MLST is a measure of genetic change for housekeeping genes, which evolve extremely slowly due to reduced tolerance for change. PFGE measures more rapid changes and the rate of gene exchange between microorganisms is only now being fully appreciated, with the advent of whole-genome sequencing (Cerdeño-Tárraga et al., 2001).

S. epidermidis strains are often considered to be part of the normal flora of human skin and as such are distributed ubiquitously (von Eiff et al., 2002). In this study, isolates either belonged to a single clonal group or were singletons. As the S. epidermidis database increases, it will be extremely interesting to see whether clonal groups also develop for the singleton isolates or there is a predominance of a single group, and whether that group is more invasive than others.

S. epidermidis epidemiology studies have been limited historically, due to the fact that $S$. epidermidis strains are often considered to be contaminants, as opposed to the disease-causing organism (Herwaldt et al., 1996), and they are usually grouped with other CoNS species. However, $S$. epidermidis infections are a rising cause of concern, due to the high distribution of methicillin resistance [some studies report up to $73 \%$ (Petinaki et al., 2001)] and their persistence on indwelling devices, often resulting in replacement of the device, which causes more trauma and is costly (Hakim et al., 2000). In this investigation, $70 \%$ of isolates were methicillinresistant. S. epidermidis is also the leading cause of nosocomial infections in neonates. Villari et al. (2000) reported an infection rate of $18.7 \%$ between 1996 and 1998 for a NICU; of these infections, $30 \%$ were due to S. epidermidis, including bloodstream infections (39.8\% of bloodstream infections), surface infections such as conjunctivitis $(29.8 \%)$ and meningitis ( $58.3 \%$ of cases).

S. epidermidis has also not received as much attention as other pathogenic micro-organisms by the various pathogen sequencing projects. The sequence of $S$. epidermidis ATCC 12228 is now complete (GenBank accession no. AE015929) and S. epidermidis RP62A is being sequenced by the Institute of Genomic Research (www.tigr.org). Our MLST analysis demonstrated that this strain is a singleton and is related only distantly to the majority of disease-causing strains, which were more similar to S. epidermidis ATCC 12228.

In this investigation, we demonstrated that a novel MLST scheme could be used to differentiate S. epidermidis isolates and act as a tool for discrimination of S. epidermidis strains from other CoNS species. As such, it will be useful for identifying divergent strains and thus will have some utility for demonstrating the target range for anti-S. epidermidis agents, such as vaccines. Work is ongoing to increase the $S$. epidermidis MLST database. MLST will not, however, be useful as a stand-alone CoNS typing method in clinical settings; it is not as discriminatory as PFGE and it is too costly and time-consuming to replace PFGE. Its utility is that it can be incorporated into polyphasic approaches for S. epidermidis epidemiology and pathogenicity studies. Peacock et al. (2002) used MLST in combination with specific pathogenicity genes to identify virulent strains of $S$. aureus. It will now be possible to do similar studies with S. epidermidis.

\section{REFERENCES}

Bannerman, T. L., Rhoden, D. L., McAllister, S. K., Miller, J. M. \& Wilson, L. A. (1997). The source of coagulase-negative staphylococci in the Endophthalmitis Vitrectomy Study. A comparison of eyelid and intraocular isolates using pulsed-field gel electrophoresis. Arch Ophthalmol 115, 357-361.

Bogado, I., Limansky, A., Sutich, E., Marchiaro, P., Marzi, M., Putero, J. \& Viale, A. (2002). Molecular characterization of methicillin-resistant coagulase-negative staphylococci from a neonatal intensive care unit. Infect Control Hosp Epidemiol 23, 447-451.

Bougnoux, M.-E., Morand, S. \& d'Enfert, C. (2002). Usefulness of multilocus sequence typing for characterization of clinical isolates of Candida albicans. J Clin Microbiol 40, 1290-1297. 
Cerdeño-Tárraga, A., Thomson, N., Holden, M., Sebaihia, M. \& Parkhill, J. (2001). Lost and found. Trends Microbiol 9, 526-527.

Dautle, M. P., Ulrich, R. L. \& Hughes, T. A. (2002). Typing and subtyping of 83 clinical isolates purified from surgically implanted silicone feeding tubes by random amplified polymorphic DNA amplification. J Clin Microbiol 40, 414-421.

Deplano, A., Vaneechoutte, M., Verschraegen, G. \& Struelens, M. J. (1997). Typing of Staphylococcus aureus and Staphylococcus epidermidis strains by PCR analysis of inter-IS256 spacer length polymorphisms. J Clin Microbiol 35, 2580-2587.

Dingle, K. E., Colles, F. M., Wareing, D. R. A. \& 7 other authors (2001). Multilocus sequence typing system for Campylobacter jejuni. J Clin Microbiol 39, 14-23.

Enright, M. C. \& Spratt, B. G. (1998). A multilocus sequence typing scheme for Streptococcus pneumoniae: identification of clones associated with serious invasive disease. Microbiology 144, 3049-3060.

Enright, M. C., Day, N. P. J., Davies, C. E., Peacock, S. J. \& Spratt, B. G. (2000). Multilocus sequence typing for characterization of methicillinresistant and methicillin-susceptible clones of Staphylococcus aureus. J Clin Microbiol 38, 1008-1015.

Geary, C., Jordens, J. Z., Richardson, J. F., Hawcroft, D. M. \& Mitchell, C. J. (1997). Epidemiological typing of coagulase-negative staphylococci from nosocomial infections. J Med Microbiol 46, 195-203.

Hakim, A., Rossi, C., Kabanda, A., Deplano, A., De Gheldre, Y. \& Struelens, M. J. (2000). Ommaya-catheter-related Staphylococcus epidermidis cerebritis and recurrent bacteremia documented by molecular typing. Eur J Clin Microbiol Infect Dis 19, 875-877.

Herwaldt, L. A., Geiss, M., Kao, C. \& Pfaller, M. A. (1996). The positive predictive value of isolating coagulase-negative staphylococci from blood cultures. Clin Infect Dis 22, 14-20.

Hu, X. \& Totake, K. (1997). Molecular typing of Staphylococcus epidermidis and other CNS with repetitive element sequence-based PCR. Microbiol Immunol 41, 933-938.

Kotetishvili, M., Stine, O. C., Kreger, A., Morris, J. G., Jr \& Sulakvelidze, A. (2002). Multilocus sequence typing for characterization of clinical and environmental Salmonella strains. J Clin Microbiol 40, 1626-1635.

Kreiswirth, B., Kornblum, J., Arbeit, R. D., Eisner, W., Maslow, J. N., McGeer, A., Low, D. E. \& Novick, R. P (1993). Evidence for a clonal origin of methicillin resistance in Staphylococcus aureus. Science 259, 227-230.

Lina, G., Etienne, J. \& Vandenesch, F. (2000). Biology and pathogenicity of staphylococci other than Staphylococcus aureus and Staphylococcus epidermidis. In Gram-positive Pathogens, pp. 450-462. Edited by V. A. Fischetti, R. P. Novick, J. J. Ferretti, D. A. Portnoy \& J. I. Rood. Washington, DC: American Society for Microbiology.

Linhardt, F., Ziebuhr, W., Meyer, P., Witte, W. \& Hacker, J. (1992). Pulsed-field gel electrophoresis of genomic restriction fragments as a tool for the epidemiological analysis of Staphylococcus aureus and coagulase-negative staphylococci. FEMS Microbiol Lett 74, 181-185.

Maiden, M. C. J., Bygraves, J. A., Feil, E. \& 10 other authors (1998). Multilocus sequence typing: a portable approach to the identification of clones within populations of pathogenic microorganisms. Proc Natl Acad Sci U S A 95, 3140-3145.

Marshall, S. A., Wilke, W. W., Pfaller, M. A. \& Jones, R. N. (1998).
Staphylococcus aureus and coagulase-negative staphylococci from blood stream infections: frequency of occurrence, antimicrobial susceptibility, and molecular $(\mathrm{mec} A)$ characterization of oxacillin resistance in the SCOPE program. Diagn Microbiol Infect Dis 30, 205-214.

Miragaia, M., Couto, I., Pereira, S. F. F. \& 7 other authors (2002). Molecular characterization of methicillin-resistant Staphylococcus epidermidis clones: evidence of geographic dissemination. J Clin Microbiol 40, 430-438.

Nahvi, M. D., Fitzgibbon, J. E., John, J. F. \& Dubin, D. T. (2001). Sequence analysis of $d r u$ regions from methicillin-resistant Staphylococcus aureus and coagulase-negative staphylococcal isolates. Microb Drug Resist 7, $1-12$.

Nallapareddy, S. R., Duh, R.-W., Singh, K. V. \& Murray, B. E. (2002). Molecular typing of selected Enterococcus faecalis isolates: pilot study using multilocus sequence typing and pulsed-field gel electrophoresis. J Clin Microbiol 40, 868-876.

Peacock, S. J., Moore, C. E., Justice, A., Kantzanou, M., Story, L., Mackie, K., O'Neill, G. \& Day, N. P. J. (2002). Virulent combinations of adhesin and toxin genes in natural populations of Staphylococcus aureus. Infect Immun 70, 4987-4996.

Petinaki, E., Kontos, F., Miriagou, V., Maniati, M., Hatzi, F. \& Maniatis, A. N. (2001). Survey of methicillin-resistant coagulase-negative staphylococci in the hospitals of central Greece. The Bacterial Resistance Study Group. Int J Antimicrob Agents 18, 563-566.

Raimundo, O., Heussler, H., Bruhn, J. B., Suntrarachun, S., Kelly, N., Deighton, M. A. \& Garland, S. M. (2002). Molecular epidemiology of coagulase-negative staphylococcal bacteraemia in a newborn intensive care unit. J Hosp Infect 51, 33-42.

Roberts, R. B., de Lencastre, A., Eisner, W., Severina, E. P., Shopsin, B., Kreiswirth, B. N. \& Tomasz, A. (1998). Molecular epidemiology of methicillin-resistant Staphylococcus aureus in 12 New York hospitals. MRSA Collaborative Study Group. J Infect Dis 178, 164-171.

Sloos, J. H., Janssen, P., van Boven, C. P. \& Dijkshoorn, L. (1998). AFLP typing of Staphylococcus epidermidis in multiple sequential blood cultures. Res Microbiol 149, 221-228.

Sloos, J. H., Dijkshoorn, L., Vogel, L. \& van Boven, C. P. A. (2000). Performance of phenotypic and genotypic methods to determine the clinical relevance of serial blood isolates of Staphylococcus epidermidis in patients with septicemia. J Clin Microbiol 38, 2488-2493.

Tenover, F. C., Arbeit, R. D., Goering, R. V., Mickelsen, P. A. Murray, B. E, Persing, D. H. \& Swaminathan, B. (1995). Interpreting chromosomal DNA restriction patterns produced by pulsed-field gel electrophoresis: criteria for bacterial strain typing. J Clin Microbiol 33, 2233-2239.

van Belkum, A., van Leeuwen, W., Kaufmann, M. E. \& 20 other authors (1998). Assessment of resolution and intercenter reproducibility of results of genotyping Staphylococcus aureus by pulsed-field gel electrophoresis of SmaI macrorestriction fragments: a multicenter study. JClin Microbiol 36, 1653-1659.

Villari, P., Sarnataro, C. \& lacuzio, L. (2000). Molecular epidemiology of Staphylococcus epidermidis in a neonatal intensive care unit over a threeyear period. J Clin Microbiol 38, 1740-1746.

von Eiff, C., Peters, G. \& Heilmann, C. (2002). Pathogenesis of infections due to coagulase-negative staphylococci. Lancet Infect Dis 2, 677-685. 